\title{
Théologiques
}

Revue interdisciplinaire d'études religieuses

Théologiques

\section{Le principe de décentralisation organisationnelle et son application en paroisse}

\section{Pierre-Alain Giffard}

Volume 27, numéro 2, 2019

Une décentralisation salutaire de l’Église catholique?

URI : https://id.erudit.org/iderudit/1069133ar

DOI : https://doi.org/10.7202/1069133ar

Aller au sommaire du numéro

Éditeur(s)

Institut d'études religieuses de l’Université de Montréal

ISSN

1188-7109 (imprimé)

1492-1413 (numérique)

Découvrir la revue

Citer cet article

Giffard, P.-A. (2019). Le principe de décentralisation organisationnelle et son application en paroisse. Théologiques, 27(2), 83-99.

https://doi.org/10.7202/1069133ar

\section{Résumé de l'article}

Cet article aborde la question de la décentralisation organisationnelle dans le cadre de la paroisse. Après avoir défini ce qu'est la décentralisation organisationnelle, et en quoi elle est déjà présente dans la vie de l’Église, l'auteur présente l'exemple d'une communauté chrétienne décentralisée pour illustrer comment la décentralisation peut aider les paroisses à être plus missionnaires et attirantes pour les nouvelles générations. L’article révèle qu'une " décentralisation salutaire " peut être exercée à différents niveaux de la vie paroissiale : les formes liturgiques, les petits groupes, la formation de leaders, la supervision des ministères, de responsables laïques et du personnel. Malgré l'importance de ce principe organisationnel et des promesses qui l'accompagnent, il est enfin rappelé que son application ne doit pas être considérée comme une fin en soi, mais comme un moyen pour mieux réaliser la mission l’Église. 


\title{
Le principe de décentralisation organisationnelle et son application en paroisse
}

\author{
Pierre-Alain GIFFARD" \\ Théologie \\ Université Saint-Paul (Ottawa, Canada)
}

Une tempête théologique, c'est bien ce que le pape François semble avoir provoqué lorsqu'il a exprimé son désir de décentralisation salutaire dans son exhortation apostolique, La joie de l'Évangiler. En Église, le sujet de la décentralisation est souvent mal compris et confus, vraisemblablement parce que ce principe fait plus partie du monde de la gestion que celui de la théologie.

À quoi le pape fait-il référence lorsqu'il parle de décentralisation? La phrase qui précède son expression décentralisation salutaire nous éclaire: «Il n'est pas opportun que le Pape remplace les Épiscopats locaux dans le discernement de toutes les problématiques qui se présentent sur leurs territoires» (François 2013, \$1). François renvoie au discernement des problématiques locales. Notons aussi que l'intervention du pape se situe dans le cadre plus large d'orientations pour une nouvelle étape évangélisatrice.

Dans cet article, j'aborderai le sujet de la décentralisation sur le plan organisationnel, administratif et bureaucratique, et non pas sur le plan juridique ou magistériel. En explorant ce que le terme veut dire dans le

Pierre-Alain Giffard est professeur de théologie ainsi que directeur de la théologie pratique à l'Université Saint-Paul d'Ottawa. Il a aussi exercé la fonction de directeur des services pastoraux de l'archidiocèse de Saint-Boniface et a été responsable de la Formation à la vie chrétienne ainsi que coordonnateur de la pastorale diocésaine dans le diocèse de Valleyfield. Sa thèse et ses intérêts portent sur le renouveau des paroisses et la croissance de l'Église. Il a récemment publié (2018) "L'annonce missionnaire et la prière du salut ", Theoforum, 48/1-2, p. 309-319 et (2019) «Leadership and Church Growth. The Case of Chuck Smith ", Great Commission Research Journal, 11/1, p. 76-93.

1. «[...] je sens la nécessité de progresser dans une "décentralisation" salutaire" (François 2013, \$16).

(C) Revue Théologiques 2019. Tout droit réservé. 
monde organisationnel et en prenant l'exemple concret d'une Église évangélique aux États-Unis, nous visons à offrir des pistes pratiques pour son application en paroisse, particulièrement dans le cadre d'une nouvelle étape évangélisatrice.

\section{La décentralisation organisationnelle}

Depuis 60 ans, la décentralisation dans le monde des organisations s'est répandue de façon fulgurante, si bien qu'elle est devenue un des principes les plus importants de la gestion moderne (Drucker et Bookspan 1990). La décentralisation peut généralement être définie comme un transfert d'autorité et de responsabilités de l'administration centrale vers les branches (ou parties) subordonnées de l'organisation. Elle vise 1) à résoudre l'inadéquation des prescriptions administratives issues de l'administration centrale avec les réalités du terrain (Affichard et Favereau 1997) et 2) à rendre l'organisation plus efficace dans l'accomplissement de sa mission.

Pour Henri Mintzberg, une structure organisationnelle peut être considérée comme centralisée «quand tous les pouvoirs de décision se situent à un seul point dans l'organisation - à la limite dans les mains d'un seul individu " (Mintzberg 2010, 173). Une structure organisationnelle est décentralisée "lorsque le pouvoir est dispersé entre de nombreuses personnes» (Mintzberg 2010, 173).

Peter Drucker, ainsi que d'autres spécialistes des organisations, explique que la décentralisation permet de mettre l'accent sur l'environnement local et de poursuivre plus efficacement les objectifs de l'organisation. Elle permet de dire: «Il y a un but mais il y a plusieurs façons de l'atteindre " (Drucker 1990, 243). Qui connaît mieux les besoins en services et en biens que les dirigeants locaux? Ils sont les plus aptes à reconnaître les moyens à prendre pour répondre aux attentes locales et à les concevoir d'une manière indigène. Une organisation décentralisée sait aussi répondre plus rapidement aux conditions locales (Drucker 1990).

En rapprochant les organisations de leurs usagers, la décentralisation des responsabilités et la création d'unités de décision de taille plus réduite autorisent un traitement personnalisé des cas, la prise en compte des réalités du terrain, une approche plus fine des situations. (Affichard et Favereau 1997, 9)

La centralisation est un mécanisme très puissant pour coordonner les décisions, mais elle tend à compromettre l'autorité et la responsabilité des dirigeants locaux et les empêche de faire un meilleur travail (Drucker 1990). 
Les sciences de la gestion montrent qu'un excès de centralisation peut aboutir à une dysfonction bureaucratique. Une organisation devenue trop rigide par une bureaucratie d'arrière-garde tend à résister à toute transformation empêchant ainsi l'adaptation des structures à l'environnement. Cette résistance bloque la croissance et l'efficacité organisationnelle. (Giffard 2012, 100)

Une organisation trop centralisée engendre une formalisation (ou standardisation) excessive des pratiques qui, elle-même, provoque l'installation de routines rigides, le maintien du statu quo, la déresponsabilisation des dirigeants locaux, l'inadéquation aux problématiques du terrain et l'insatisfaction des bénéficiaires.

Malgré ses avantages, l'application de la décentralisation n'est pas toujours aisée et rencontre des défis: il y a notamment celui de tenir ensemble diversité organisationnelle et unité. Si la décentralisation permet d'éviter un contrôle abusif qui nuit à l'efficacité, trop de décentralisation et d'indépendance peuvent conduire à des divisions qui nuisent à l'atteindre de l'objectif global. Aussi, plus la décentralisation est grande, plus il y a d'incertitudes pour l'administration centrale quant à ces effets (Affichard et Favereau 1997).

Même s'il y a certains défis à surmonter, les grandes organisations ont avantage à se décentraliser, parce qu'un seul centre ne peut discerner toutes les décisions à prendre au niveau local. L'information du terrain ne peut parvenir adéquatement jusqu'au centre. Et même quand elle y parvient, le centre n'a pas les capacités cognitives pour interpréter l'ensemble des données en provenance du terrain.

Il est parfois tout simplement impossible à l'information nécessaire d'arriver à ce centre. Peut-être une trop grande partie de l'information est floue, difficile à transmettre. Quelquefois, l'information peut être transmise au centre, mais un manque de capacité cognitive empêche qu'elle n'y soit comprise. [...] Les cadres dirigeants, qui ont l'autorité nécessaire pour concevoir la structure, constatent que des erreurs sont commises aux niveaux inférieurs; et ils pensent pouvoir faire mieux, soit parce qu'ils s'estiment eux-mêmes plus compétents, soit parce qu'ils pensent pouvoir mieux coordonner les décisions. Malheureusement, ce comportement conduit inéluctablement, dans des situations complexes, à un état connu sous le nom de surcharge d'information: plus le centre essaie de recevoir d'informations, moins il en a en réalité. En d'autres termes, passé un certain point, les cadres ne peuvent ni être meilleurs, ni mieux coordonnés. Ils feraient mieux de laisser certains pouvoirs de décision à d'autres qui disposent du temps et de la capacité nécessaires au traitement des informations. (Mintzberg 1982, 173) 
La décentralisation est aussi liée à la motivation des membres d'une organisation. Les personnes créatives et intelligentes sont plus motivées lorsqu'elles ont plus de marge de manœuvre et de pouvoir de décision: «Une telle motivation est cruciale dans les travaux de nature professionnelle $[. .$.$] . Cette motivation est aussi un facteur clé pour les postes d'enca-$ drement, et un certain degré de décentralisation est donc justifié dans la ligne hiérarchique» (Mintzberg 1982, 176).

\section{La décentralisation et le peuple de Dieu}

L'Église, en tant que réalité à la fois humaine et spirituelle, n'échappe pas aux règles des organisations en général. En regardant de près, on se rend compte que la décentralisation n'est pas nouvelle dans l'Église. Dans son discours au Collège des cardinaux le 24 juin 1971, le pape Paul VI avait fait l'éloge de la "décentralisation, opérée pendant et après le Concile, concernant de multiples affaires réservées jusqu'alors aux dicastères romains» (Paul VI 1971).

Lors de la $\mathrm{V}^{\text {e }}$ Conférence générale de l'épiscopat latino-américain à Aparecida, les évêques latino-américains avaient proposé une décentralisation des services ecclésiaux afin de permettre à un plus grand nombre d'agents pastoraux de s'impliquer dans la mission (CGELAC 2007, 225).

En 2001, l'Instrumentum Laboris du Synode des évêques à Rome exprimait le souhait d'une décentralisation administrative des responsabilités de gouvernement de l'évêque. Et cette même année, lors de la $\mathrm{X}^{\mathrm{e}}$ Assemblée ordinaire du Synode des évêques, il avait été suggéré d'étudier la question de la décentralisation au niveau des rapports entre l'évêque et la Curie romaine à la lumière du principe de subsidiarité (Jean-Paul II 2003).

La subsidiarité, qui justifie la décentralisation, est un principe de la doctrine sociale de l'Église stipulant qu'«il revient à chaque degré d'autorité d'exercer toutes les attributions qui lui sont propres sans avoir besoin de recourir à une autorité de plus grande envergure" (D'Onorio 1995, 12).

Les premières traces de ce principe remontent à Aristote: décrivant la société comme un ensemble de groupes emboîtés les uns dans les autres (famille, village, cité), il légitime la notion d'autorité par la notion de suffisance et d'insuffisance. L'intervention de l'échelon supérieur dans les

2. La décentralisation bureaucratique et administrative est considérée par l'Église comme une application directe du principe de subsidiarité. 
compétences de l'échelon subordonné ne se justifie qu'à titre supplétif ou subsidiaire, c'est-à-dire en cas de carence voire de défaillance du subordonné.

On parle de subsidiarité descendante (ou négative) quand on se réfère au pouvoir supérieur qui doit laisser autant d'attributions qu'il est nécessaire aux pouvoirs inférieurs; et de subsidiarité ascendante (ou positive) quand on se réfère au pouvoir supérieur qui doit agir en suppléance des échelons inférieurs quand ils se trouvent incompétents ou dépassés.

La subsidiarité peut autant s'appliquer au sein des collectivités locales que dans les établissements publics et les entreprises privées. Il s'agit d'un principe universel qui dépasse la simple organisation de la vie économique ou politique. Il est un principe de droit naturel lié aux libertés individuelles et collectives qui garantit l'épanouissement de la personne: il embrasse la totalité de la vie sociale.

Des traces de décentralisation et de subsidiarité peuvent être trouvées dans le live de l'Exode. Jéthro, voyant tout ce que Moïse faisait pour le peuple, lui dit:

«Comment t'y prends-tu pour traiter seul les affaires du peuple? Pourquoi sièges-tu seul alors que tout le peuple se tient auprès de toi du matin au soir ?» "[...] Choisis-toi parmi tout le peuple des hommes capables, craignant Dieu, sûrs, incorruptibles, et établis-les sur eux comme chefs de milliers, chefs de centaines, chefs de cinquantaines et chefs de dizaines. Ils jugeront le peuple en tout temps. Toute affaire importante, ils te la déféreront et toute affaire mineure, ils la jugeront eux-mêmes. Allège ainsi ta charge et qu'ils la portent avec toi.» (Ex 18, 1-27)

Moïse suivit les conseils de son beau-père et opéra une authentique décentralisation, c'est-à-dire un transfert de fonctions administratives et une délégation de pouvoir de décision.

Aussi l'Ancien Testament nous présente un Dieu qui «fait alliance avec Israël sans absorber ni fusionner en aucune façon les différentes tribus qui gardaient chacune leur autonomie, ainsi les hommes sont-ils appelés à vivre en bonne entente, avec la nécessité d'un pacte global, mais sans toutefois dissoudre les liens locaux et les prérogatives des échelons inférieurs » (Commission sources bibliques et théologiques 2016, 18).

Jésus lui-même ne se vit pas l'unique acteur et responsable de la mission que le Père lui avait confiée, il partagea avec d'autres sa mission, son autorité et son pouvoir en déléguant même sa faculté de guérir, de pardonner et d'opérer des miracles (Lc 9,1-2; 10,19; Jn 14,12; 20,23; Ac 1,8). 


\section{L'exemple d'Amplify Church}

Prenons maintenant le cas d'Amplify Church, une Église évangélique de type paroissial qui contient plusieurs éléments de décentralisation. Trente ans après sa fondation à Pittsburgh aux États-Unis, ses membres ne pouvaient que constater que l'Église était en train de mourir. L'édifice était en piètre état à cause du manque d'entretien. Les systèmes de chauffage ne fonctionnaient plus, le stationnement était une mare de graviers et de boue et le toit coulait sur le piano.

Le conseil d'administration de l'Église décida donc de reprendre contact avec Lee Kricher, qui avait fondé l'Église sous le nom de Pittsburgh East Full Gospel Church ${ }^{3}$. Non sans humour, ce dernier constata que cette communauté chrétienne de 200 personnes était devenue «une des Églises de la ville qui mourrait le plus rapidement» (Kricher 2016, 41, nous traduisons).

Malgré la taille du défi qui l'attendait, il accepta l'offre de revenir diriger l'Église. Dix ans après, grâce à d'importants changements et à l'application du principe de décentralisation, presque 2000 personnes assistent aux célébrations du dimanche. Dans ses efforts de renouveau, cette communauté chrétienne s'est étendue sur deux autres sites: un campus pour les familles avec de jeunes enfants dans l'Indiana et une ancienne boîte de nuit convertie en Centre pour les adolescents et jeunes adultes.

Le pasteur rebaptisa l'église Amplify Church et voulut que les responsabilités soient partagées et synchronisées autour d'un but précis: celui de devenir une Église de la nouvelle génération. Il décentralisa son pouvoir en créant une équipe de direction. Pour en faire partie, les membres devaient avoir les caractéristiques suivantes: une position d'influence dans l'Église, de la crédibilité, de l'expertise et du leadership. Certaines personnes étaient à éviter : celles dont l'égo était trop grand et dont le comportement négatif pouvait compromettre tout progrès (Kricher 2016). Selon lui, choisir les bonnes personnes était crucial, tout comme le fait de demander aux personnes en désaccord avec le nouveau but de partir.

Pour ce pasteur, chercher à être une Église de la nouvelle génération signifiait tout faire pour que l'âge moyen de l'Église corresponde à l'âge moyen de la communauté dans laquelle elle existe. Il s'agit de «penser en termes de renouvellement continuel » (Kricher 2016, 21, 131, nous tradui-

3. Lee Kricher avait quitté son poste de pasteur en 1990 pour retourner dans le monde des affaires. 
sons). Aux États-Unis, où de plus en plus de personnes, particulièrement les jeunes, ne fréquentent pas d'églises, cela représentait tout un défi.

Pour aider l'équipe de direction à saisir la vision, le pasteur emmena ses membres visiter d'autres églises pleines de vie et remplies de jeunes. Le pasteur fit aussi lire à l'équipe de direction des livres sur la croissance et le renouvellement des églises de type paroissial. Ces expériences et ces lectures contribuèrent à transformer la mentalité de la communauté chrétienne et à entrer dans une nouvelle étape évangélisatrice.

Changer les mentalités dans l'Église fut en fait la première étape d'une stratégie en cinq points:

1) Adopter une nouvelle mentalité (être prêt à changer pour devenir une Église de la nouvelle génération)

2) Discerner l'essentiel

3) Réduire les distractions

4) Rechercher l'excellence

5) Bâtir une culture de mentorat

Pour que tous et toutes travaillent de concert à devenir une Église de la nouvelle génération, un énoncé de vision fut proposé par le pasteur et accepté par l'équipe de direction: "Amener le plus de personnes que possible dans une relation avec Jésus-Christ et un cheminement de croissance spirituelle» (Kricher 2016, 61, nous traduisons).

L'équipe de direction chercha ensuite à déterminer des orientations pastorales qui permettraient de réaliser de la vision. Elle chercha aussi à éliminer autant que possible les programmes, les ministères et les pratiques en place qui n'étaient pas en harmonie avec l'énoncé. Il s'agissait de rediriger le temps, les énergies et les ressources de l'Église sur ce qui était essentiel.

Une attention toute particulière devait être portée à l'excellence sur tous les plans, particulièrement celui des célébrations, de la prédication et de la musique. Rechercher l'excellence, pour Lee Kircher, ne veut pas dire être en concurrence avec d'autres Églises, mais plutôt « réduire la distance qui existe entre ce que l'Église accomplit présentement et ce qu'elle est capable d'accomplir afin d'atteindre son plein potentiel» (Kricher 2016, 105 , nous traduisons).

C'est aussi une culture de mentorat qui contribua à la décentralisation de l'Église. Le pasteur mit en place un système permettant d'intégrer de nouveaux membres dans des rôles de leadership et de responsabilité; chaque leader était appelé à coacher au moins deux mentorés. Le but était 
que la communauté chrétienne non seulement devienne, mais continue d'être, une Église de la nouvelle génération.

L'équipe de direction se donna trois priorités: la création de petits groupes, la conception de célébrations exceptionnelles et le développement de ministères auprès des enfants et des jeunes. Les célébrations adoptèrent un style de musique contemporain, et les participants un code vestimentaire décontracté. À l'exception des valeurs fondamentales ou de la foi de l'Église, tout a été réfléchi et remis en question pour réaliser la vision: «Sans compromettre notre foi ou nos valeurs de base, nous ferons tout ce qu'il faut pour rejoindre la nouvelle génération avec le message de JésusChrist qui transforme nos vies» (Kricher 2016, 56, nous traduisons).

Un des changements apportés à l'Église est l'augmentation progressive du pouvoir confié aux bénévoles en fonction de leurs responsabilités (empowerment). Le pasteur explique que son style de leadership a consciemment changé avec le temps. Il estimait en effet qu'un leadership plus directif était nécessaire au début, afin d'apporter les premiers changements, et que par la suite il lui fallait adopter un leadership partagé (ou participatif), permettant aux autres responsables de prendre plus de décisions.

L'équipe de direction décida d'éliminer les célébrations en semaine pour se concentrer sur celles du week-end et réduisit la durée de celles-ci de 90 à 65 minutes. Tous les membres étaient invités à se joindre personnellement à des petits groupes d'études bibliques.

La petite chapelle de l'église fut transformée en un théâtre pour enfants avec un système de son et de lumière de qualité professionnelle. Beaucoup de temps et d'argent furent investis dans la musique et les répétitions. Le pasteur explique que la prière a aussi joué un rôle essentiel dans leur processus de transformation missionnaire: "La prière était certainement l'un des principaux facteurs de la revitalisation de notre Église. [...] Tout changement et toute revitalisation durable commencent lorsque nous reconnaissons notre dépendance de Dieu et lui demandons de faire ce que lui seul peut faire» (Kricher 2016, 24, nous traduisons).

Enfin, l'évangélisation auprès de ceux et celles qui ne connaissent pas Jésus ou qui ne le suivent pas ainsi que la croissance spirituelle des membres ont été considérées comme des priorités. Dans ce sens, les bénévoles étaient encouragés à inviter régulièrement de nouvelles personnes à l'église, particulièrement celles de leur groupe d'âge.

Si cette Église a réussi à se décentraliser et à entrer dans une nouvelle étape évangélisatrice, les changements ne se sont pas réalisés sans résistances et conflits. Parmi les plus sérieux furent ceux provoqués par l'élimi- 
nation de programmes et d'activités qui ne s'alignaient pas avec la nouvelle vision. Pour le pasteur, il ne s'agissait pas de faire beaucoup de bonnes choses, mais de faire les bonnes choses. Hélas, certains membres ont décidé de quitter l'église à cause de ces changements.

En bref, ce qui assura la revitalisation et la décentralisation de cette communauté chrétienne fut la décision de devenir une Église pour la nouvelle génération, la communication de cette nouvelle vision, la création d'une équipe de direction, une culture de mentorat et de responsabilisation des laïcs face à la mission, la création d'activités et de programmes pour réaliser la vision, une planification rigoureuse, les efforts de communication pour changer la culture de la communauté chrétienne, l'élimination des activités qui n'étaient pas en ligne avec la nouvelle vision, l'implication des membres de l'Église, l'innovation et la conformité à la culture de la nouvelle génération, la recherche d'excellence, la création de petits groupes, les activités pour les enfants et les jeunes, la prière et l'évangélisation par invitation.

\section{Décentralisation et paroisses}

L'expérience d'Amplify Church permet de mieux comprendre la valeur de la décentralisation appliquée à la paroisse et présente plusieurs façons de la mettre en œuvre. Certes, en tant qu'Église non confessionnelle, Amplify Church n'est pas soumise au contrôle d'un pouvoir central ${ }^{4}$ qui oblige ses paroisses à suivre des prescriptions liturgiques rigides. Cette liberté donne la possibilité d'adapter plus facilement les célébrations à la culture des nouvelles générations. Toutefois et malgré les contraintes institutionnelles de l'Église catholique, le pape actuel encourage diverses formes de paroisses ainsi que la créativité afin de rompre avec des schémas ennuyeux dans lesquels nous avons enfermé nos communautés chrétiennes.

Dans sa venue, [le Christ] a porté avec lui toute nouveauté. Il peut toujours, avec sa nouveauté, renouveler notre vie et notre communauté, et même si la proposition chrétienne traverse des époques d'obscurité et de faiblesse ecclésiales, elle ne vieillit jamais. Jésus Christ peut aussi rompre les schémas ennuyeux dans lesquels nous prétendons l'enfermer et il nous surprend avec sa constante créativité divine. Chaque fois que nous cherchons à revenir à la source pour récupérer la fraîcheur originale de l'Évangile, surgissent de nouvelles voies, des méthodes créatives, d'autres formes d'expression, des

4. C'est le cas par exemple pour des paroisses catholiques ou anglicanes. 
signes plus éloquents, des paroles chargées de sens renouvelé pour le monde d'aujourd'hui. En réalité, toute action évangélisatrice authentique est toujours «nouvelle». (François 2013, $\mathbb{S} 11$, nous soulignons)

La paroisse n'est pas une structure caduque; précisément parce qu'elle a une grande plasticité, elle peut prendre des formes très diverses qui demandent la docilité et la créativité missionnaire du pasteur et de la communauté. (François 2013, \$28)

En outre, dans chaque pays ou région, peuvent être cherchées des solutions plus inculturées, attentives aux traditions et aux défis locaux. (François 2016, \$3)

Dans leur désir d'entrer dans une nouvelle étape évangélisatrice, les responsables d'Amplify Church ont cherché à ce que les personnes invitées et les personnes non chrétiennes se sentent à l'aise lors des célébrations; que celles-ci ne soient pas perdues dans des formes liturgiques incompréhensibles pour des non-initiés. Les célébrations ont été conçues pour favoriser une rencontre avec le Christ et une croissance dans la foi, tout en faisant en sorte que l'ambiance, le genre de musique et un style vestimentaire décontracté rejoignent et attirent les nouvelles générations.

Une décentralisation des formes liturgiques ${ }^{5}$ permettrait aux paroisses d'être plus créatives et de bâtir des célébrations plus attirantes pour les jeunes et les jeunes adultes. Le pape François invite lui-même les chrétiens «à être audacieux et créatifs pour repenser les objectifs, les structures, le style et les méthodes évangélisatrices de leurs communautés chrétiennes » (François 2013, \$33).

Par la mise sur pied d'une équipe de direction, Amplify Church donne aussi l'exemple d'une décentralisation qu'une paroisse peut effectuer au niveau de l'exercice du pouvoir. La gestion et la revitalisation d'une paroisse sont en effet des tâches trop lourdes et trop complexes pour être seulement pensées et organisées par le pasteur. Aussi, lorsqu'un prêtre est affecté ailleurs et remplacé, tout ne tombe pas à l'eau, l'équipe peut assurer la continuation des projets entrepris et des buts poursuivis.

C'est aussi la création d'une structure composée de petits groupes, animés par des responsables laïcs, qui montre comment une paroisse peut

5. Ici, lorsque qu'on parle d'une décentralisation des formes liturgiques, il est entendu une plus grande souplesse de la part des autorités centrales de l'Église pour donner aux paroisses la possibilité de célébrer les sacrements de façon plus adaptée à la culture des nouvelles générations. Les liturgies actuelles sont obligatoirement lues, manquant ainsi de spontanéité. Cela a pour conséquence de rendre les célébrations ennuyantes et figées. 
se décentraliser. C'est ce que fit l'Église Amplify Church: «En plus des célébrations de fin de semaine, nous nous sommes concentrés sur la création d'un solide programme pour les petits groupes. Nous avons senti que les petits groupes étaient essentiels pour aider les gens à construire une communauté et à vivre une communion de plus en plus profonde avec Jésus-Christ» (Kricher 2016, 68, nous traduisons). Bien que la forme de cette structure ne soit pas nouvelle dans l'Église Catholique, celle-ci n'est pas très présente dans ses paroisses ${ }^{6}$. Une structure de petits groupes permet de mieux incarner la réalité de l'Église-communion et d'engager les membres de la communauté chrétienne dans la mission. Les petits groupes ecclésiaux sont, selon Donald McGavran ${ }^{7}$, un des éléments qui assure la croissance d'une Église. Les petits groupes «permettent à une petite église de grandir considérablement» (McGavran 1980, 217, nous traduisons).

Les recherches en management montrent que les empires industriels américains doivent leur développement parfois spectaculaire aux vertus de structures de type décentralisé. Et c'est bien le passage à une structure ecclésiale décentralisée par petits groupes qui a permis à l'Église du plein Évangile de Yoido de croître considérablement, passant en moins de 50 ans de quelques personnes à 800000 membres. Son pasteur s'exprime ainsi : "J'ai expliqué précédemment que la croissance de notre Église est basée sur les buts fixés et sur l'implantation de cellules de maison» (Cho 1989, 83).

Les petits groupes permettent aussi aux paroissiens d'exercer leurs dons. Personne n'est passif, tout le monde est impliqué. C'est ce type de structure qui a rendu possible le développement extraordinaire de l’Église International Charismatic Mission, dirigée par Cesar Castellanos. Le but de ce pasteur est que chaque membre de son Église devienne un responsable de petit groupe (Comiskey 2002).

À la manière d'Amplify Church, une paroisse peut aussi décentraliser la formation des leaders. Ce sont alors des laïcs, et non le pasteur, qui

6. Ces 30 dernières années, le modèle des petits groupes en paroisse dans les Églises plus traditionnelles, tel que les cellules paroissiales d'évangélisation, trouve son origine dans la megachurch pentecôtiste coréenne: Yoido Full Gospel Church (voir la thèse de doctorat de Pierre-Alain Giffard: De la croissance numérique à la croissance intégrale: un modèle de mission pour l'Église locale [2001]).

7. McGavran est considéré, dans le milieu protestant évangélique, comme le fondateur du Mouvement de la croissance de l'Église. Ce mouvement, comme son nom le suggère, s'intéresse aux facteurs qui favorisent la croissance des communautés chrétiennes. Il est composé d'un ensemble de praticiens et de théologiens qui étudient les éléments favorisant le développement des Églises. 
forment de nouveaux membres, permettant ainsi de remplacer des leaders laïcs existants ou d'en former d'autres. Cette délégation est essentielle pour assurer la continuation et la multiplication des services sans épuiser le pasteur. Elle est aussi indispensable pour remplacer les responsables qui quittent la paroisse ou pour fournir de nouveaux leaders au fur et à mesure que l’Église grandit.

Dans une paroisse, explique James Mallon, la décentralisation de la supervision des ministères laïcs est nécessaire. Le pasteur ne doit pas assurer l'entière supervision des ministères, des responsables laïcs et du personnel: «Dans les très grandes paroisses, le pasteur rencontrera directement le personnel et les responsables de ministères qui supervisent eux-mêmes d'autres membres du personnel et des responsables de ministères " (Mallon 2014, 262). Si cette décentralisation ne se fait pas, la vie de la paroisse va stagner car le pasteur n'a pas à lui seul suffisamment d'énergie et de temps pour tout accomplir.

La décentralisation peut aussi s'exercer au niveau de la pastorale sociale. Grâce aux petits groupes, les membres de la paroisse peuvent réfléchir ensemble aux talents que Dieu leur a donnés et concevoir des activités pour intervenir auprès des personnes souffrantes de leur milieu: «[...] d'une certaine façon, nos petits groupes sont missionnaires dans le sens où ils sont les mains et les pieds du Christ à l'extérieur de notre église » (Kricher 2016, 71, nous traduisons).

Finalement, et de façon métaphorique, l'expérience d'Amplify Church invite les responsables d'Église à "décentraliser» leur attention — souvent portée presque uniquement sur la célébration des sacrements - pour la fixer sur la mission de faire des disciples et sur la création des activités pastorales qui la rendent possible. La mission du prêtre n'est pas seulement de célébrer les sacrements mais aussi d'organiser la communauté chrétienne pour que celle-ci fasse des disciples de Jésus. Ceci est la mission centrale de l'Église et une des caractéristiques principales des paroisses missionnaires dans le monde: «Allez donc, de toutes les nations faites des disciples, les baptisant au nom du Père et du Fils et du Saint-Esprit, et leur apprenant à observer tout ce que je vous ai prescrit» (Mt 28,19-20).

\section{Conclusion}

En quoi la décentralisation peut-elle être salutaire pour l'Église? Nous avons vu que la décentralisation permet d'abord de mettre l'accent sur l'environnement, de mieux tenir compte des réalités du milieu et d'être plus 
efficace dans la poursuite de ses buts. Appliquée à une Église de type paroissial, elle permet plus de souplesse, d'adaptation à la culture des nouvelles générations, de créativité et de diversité dans les célébrations.

Au niveau du leadership et de la prise de décision, et grâce à la mise sur pied d'une équipe de direction, la décentralisation peut amener plus de stabilité et de continuité dans la réalisation de projets à moyens et à long terme ${ }^{8}$. Par le biais d'une telle équipe, les dirigeants peuvent partager leur pouvoir et en arriver à planifier avec les laïcs la réalisation des buts et des objectifs paroissiaux.

La décentralisation appliquée à la paroisse facilite la formation des leaders et la supervision des ministères laïcs. Grâce à la mise en place de petits groupes, elle permet de mieux vivre la dimension d'accueil, de fraternité et de justice sociale. Enfin, les laïcs sont plus motivés et peuvent plus facilement exercer leurs dons.

C'est probablement sur le plan liturgique que le besoin de la décentralisation se fait le plus sentir aujourd'hui. En effet, les changements socioculturels se produisent de façon si accélérée que les nouvelles générations ne se sentent pas attirées par la forme rigide et monotone des célébrations actuelles. ${ }^{9}$

Grâce à plus de décentralisation de la part des autorités centrales de l'Église, les évêques et les prêtres diocésains ainsi que les laïcs œuvrant sur le terrain pourraient concevoir des célébrations plus intéressantes et mieux adaptées en saisissant les éléments de la culture locale et particulièrement celle des nouvelles générations. Cela répondrait d'ailleurs aux recommandations du concile Vatican II qui invite les Églises locales à «joindre les éléments traditionnels de l'Église Universelle à leur culture propre, pour accroittre la vie du Corps mystique par des échanges mutuels » (Ad Gentes $1965, \$ 19)$.

Le problème des Églises trop centralisées est qu'elles fonctionnent sous un mode autocratique, distant du terrain et ne laissent que très peu de place à la créativité et à la participation des paroisses dans les décisions. Cela empêche, entre autres, l'adaptation des célébrations et étouffe le désir de s'impliquer des membres. Quand les pratiques, les structures et les liturgies des paroisses sont définies de façon si formelle, les pasteurs peuvent

8. C'est d'autant plus important lorsque les prêtres d'un diocèse sont peu nombreux, assument la responsabilité de plusieurs communautés chrétiennes et changent d'affectation souvent.

9. Nous faisons référence ici aux paroisses plus traditionnelles, comme les Églises anglicanes, catholiques et luthériennes. 
être conduits à concevoir leur mission comme celle d'une simple conformité aux règles. Ils démontrent leur fidélité à l'organisation plus par leur respect des prescriptions que par la créativité liturgique pour mieux accomplir la mission de l’Église (Is 43,19; Ap 21,5).

Si elle ne se décentralise pas davantage, l'Église pourrait se retrouver dans une situation d'enculturation comme après le concile de Trente. Cette période fut celle d'une grande uniformisation qui toucha la liturgie, le langage théologique et la formation des clercs et des religieux.

La formation uniforme du clergé dans toutes les parties du monde eut deux conséquences: 1) elle accentua la séparation entre le clergé (et les religieux) formée sur les bases d'une pensée philosophique médiévale et un laïcat formé selon les critères de la science moderne; 2) elle ignora le contexte culturel et les richesses culturelles des pays évangélisés. En conséquence, la même forme de liturgie, de réflexion théologique, d'enseignement catéchétique et d'organisation ecclésiale était imposée aux vieilles cultures d'Asie comme aux peuples aborigènes d'Amérique du Sud. Les tentatives de missionnaires comme Ricci et De Nobili d'évangéliser la Chine et l'Inde à partir des cultures locales furent rapidement rejetées (Veilleux 2010).

Aujourd'hui, la mission n'est plus dans les pays lointains mais bien en Occident, où il y a nécessité pour les paroisses d'adapter leurs activités à la culture et aux besoins de leur milieu. L'expérience montre qu'en matière de fréquentation les Églises évangéliques, ou non confessionnelles, comme Amplify savent attirer les nouvelles générations par des célébrations culturellement adaptées ${ }^{10}$.

Enfin, lorsque l'on parle de décentralisation, il est essentiel de ne pas oublier que la forme organisationnelle ne doit pas devenir plus importante que la fonction de l'organisation. À ce sujet, Peter Drucker explique que ce qui est premier n'est pas de choisir la structure, mais de se demander: "quelle est notre mission?» (Drucker 2008). Une fois cette question répondue, la structure peut être choisie. Améliorer la structure organisationnelle - par une plus grande décentralisation - permet toujours d'améliorer l'atteinte des objectifs de l'organisation. Adopter une bonne structure organisationnelle n'est pas la solution miracle, toutefois cela est

10. Les personnes qui font l'expérience des célébrations des Églises évangéliques diront que celles-ci sont plus vivantes et spontanées que celles des Églises plus traditionnelles. Les jeunes et les jeunes adultes qui forment une bonne partie de l'assemblée s'y retrouvent «culturellement». Ils s'habillent selon leur habitude, le style de musique est contemporain et l'architecture même des églises est moderne et fonctionnelle. 
nécessaire afin d'établir de bonnes fondations. Sans celles-ci, les efforts dans les autres dimensions de la gestion seront étouffés (Drucker 1990).

Le mandat de faire des disciples est au cœur de la mission de l'Église. Et, à notre avis, c'est en fonction de ce mandat, et de l'efficacité à le remplir, qu'il est nécessaire de penser à la décentralisation de l'Église. C'est à partir d'un tel paradigme que le principe de décentralisation prendra tout son sens et pourra être réellement salutaire.

\section{Références}

Affichard, J., et O. Favereau (1997), Décentralisation des organisations et problèmes de coordination. Les principaux cadres d'analyse, Paris, L'Harmattan.

Castillo Lara, R. J. (1995), "La subsidiarité dans l'Église», dans J.-B. D’onorio, La subsidiarité, Saint-Cénéré, Éditions Téqui.

Сно, P. Y. (1989), Les cellules de maison et la vie de l'Église, Miami, Ed Vida.

Comiskey, J. (2002), Home Cell Group Explosion. How Your Small Group Can Grow and Multiply, Houston, Touch Publications.

Commission sources bibliques et théologiques (2016), La subsidiarité. Comprendre et mettre en cuvre le principe de subsidiarité dans l'entreprise, Collection La pensée sociale chrétienne, Publication des EDC, Extrait de <www.lesedc.org/publication/le-cahier-subsidiarite/>.

Concile Vatican II (1965), Décret sur l'activité missionnaire de l'Église Ad Gentes, Extrait de <www.vatican.va/archive/hist_councils/ii_vatican_ council/documents/vat-ii_decree_19651207_ad-gentes_fr.html>.

CONFÉRENCE GÉNÉRALE DE L'ÉPISCOPAT LATINO-AMÉRICAIN ET DES CARAÏ̈Bes (CGELAC) (2007), Disciples et missionnaires de Jésus-Christ pour que nos peuples aient la vie en lui, $\mathrm{V}^{\mathrm{e}}$ conférence à Aparecida, Document final, Extrait de: <www.celam.org/aparecida/Frances.pdf $>$.

D’Onorio, J.-B. (1995), «La subsidiarité, analyse d'un concept », La subsidiarité. De la théorie à la pratique, Saint-Cénéré, Éditions Téqui.

Drucker, P. F., et M. Bookspan (1990), The practice of management, New York, HarperCollins.

Drucker, P. F., et Frances H. L. Institute (2008), The Five Most Important Questions You Will Ever Ask About Your Organization, San Francisco, Jossey-Bass. 
Fischer, R. J. (1999), "Délégation de pouvoir et décentralisation de la gestion forestière en Asie et dans le Pacifique. Différents aspects et problèmes », Unasylva, 50/199, Extrait de <www.fao.org/docrep/x3030f/ x3030f03.htm>.

FrançoIs (2013), Evangelii Gaudium, Extrait de: <w2.vatican.va/content/ francesco/fr/apost_exhortations/documents/papa-francesco_esortazioneap_20131124_evangelii-gaudium.html>.

(2016), Amoris Lotitia, Extrait de: <w2.vatican.va/content/francesco/fr/apost_exhortations/documents/papa-francesco_esortazioneap_20160319_amoris-laetitia.html>.

GifFARD, P.-A, (2001), De la croissance numérique à la croissance intégrale: Un modèle de mission pour l'Église locale, thèse de doctorat pratique, Université de Montréal, Canada.

(2012), La croissance de l'Église. Outils et réflexions pour dynamiser nos paroisses, Québec, Éditions des Béatitudes.

Jay, A. (1974), Decentralization in Organizations, working paper, International institute of Management, West Berlin.

Jean-Paul II (2003), Exhortation Apostolique Post-synodale Pastores Gregis, Extrait de <w2.vatican.va/content/john-paul-ii/fr/apost_ exhortations/documents/hf_jp-ii_exh_20031016_pastores-gregis.html>.

Kricher, L. (2016), For a New Generation A Practical Guide for Revitalizing Your Church. Grand Rapids, Zondervan.

Mallon, J. (2014), Divine Renovation. From a Maintenance to a Missional Parish, Toronto, Novalis publishing Inc.

McGavran, D. (1980), Understanding Church Growth, Revised ed. by C. Peter Wagner, Grand Rapids, B. Eerdmans Publishing Co.

Mintzberg, H. (2010), Structure et dynamique des organisations, Les Éditions d'Organisations, Paris.

PAul VI (1971), «Discours au collège des cardinaux du 24 Juin 1971 », Extrait de : <www.clerus.org/bibliaclerusonline/pt/kdm.htm>.

Synode des ÉvÊQues (2001), Xe Assemblée Générale Ordinaire, L'Évêque, serviteur de l'Évangile de Jésus-Christ pour l'espérance du monde, Instrumentum Laboris, Vatican, Libreria Editrice Vaticana.

Veilleux, A. (2010, février), Qu'arrive-t-il à l'Église aujourd'bui?, Conférence donnée à l'Université de Louvain-la-Neuve, à Louvain, le 8 février 2010, et à Bruxelles, le 9 février, dans le cadre d'un cycle de quatre conférences organisées par la Fondation Sedes Sapientiae et la faculté de Théologie de l'UCL. Repéré à <www.scourmont.be/Armand/ writings/louvain-2010.htm>. 
Witzel, M. (2010²) [2005], The Encyclopedia of the History of American Management, London, Continuum. Extrait de: <www.oxfordreference. $\mathrm{com} / \mathrm{view} / 10.1093 / \mathrm{acref} / 9780199754687.001 .0001 / \mathrm{acref}-$ 9780199754687-e-168? rskey=Z7GylI\&result=9>.

\section{Résumé}

Cet article aborde la question de la décentralisation organisationnelle dans le cadre de la paroisse. Après avoir défini ce qu'est la décentralisation organisationnelle, et en quoi elle est déjà présente dans la vie de l'Église, l'auteur présente l'exemple d'une communauté chrétienne décentralisée pour illustrer comment la décentralisation peut aider les paroisses à être plus missionnaires et attirantes pour les nouvelles générations. L'article révèle qu'une « décentralisation salutaire » peut être exercée à différents niveaux de la vie paroissiale: les formes liturgiques, les petits groupes, la formation de leaders, la supervision des ministères, de responsables laïques et du personnel. Malgré l'importance de ce principe organisationnel et des promesses qui l'accompagnent, il est enfin rappelé que son application ne doit pas être considérée comme une fin en soi, mais comme un moyen pour mieux réaliser la mission l'Église.

\section{Abstract}

This article discusses the topic of organizational decentralization applied to the parish. Having defined what organizational decentralization is and how it is already present in the life of the Church, the example of a decentralized parish is taken to show how decentralization can help parishes to be more missional and attractive to the new generations. The article reveals how decentralization can be applied to different levels of parish life: liturgical forms, small groups, leadership training, oversight of ministries, lay leaders, and staff. Despite the importance of this principle and of the promises it carries, it is reminded that its application should not be considered as an end in itself but as a means to better accomplish the mission of the Church. 